\title{
REHABILITATION OF THE AMPUTEE
}

\author{
S J D Chadwick, John H N Wolfe
}

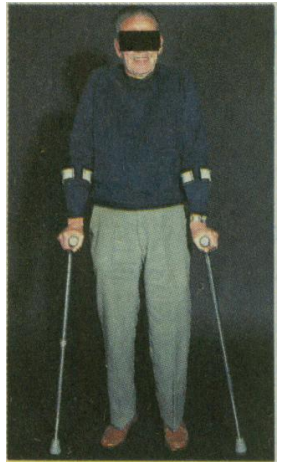

A successful above knee amputation. The patient was walking on crutches within 10 days of operation. He had run eight miles a day until acute thrombosis of a popliteal aneurysm produced irreversible ischaemia.

A bilateral above knee amputee who was confined to a wheelchair but remained motivated and relatively independent.

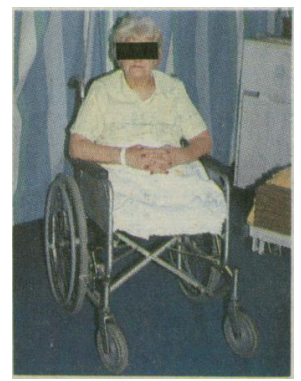

\section{Process of rehabilitation}

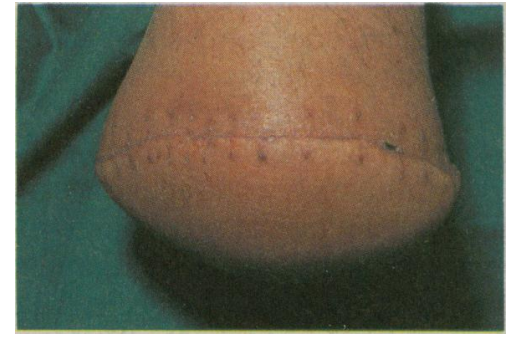

A well healed below knee amputation stump. The muscularity of the leg has not been adequately accounted for, resulting in a bulky stump that is difficult to fit with a prosthesis.

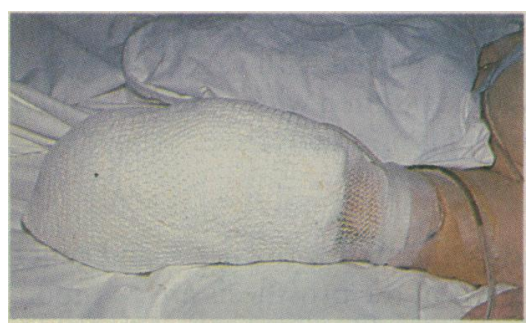

The ideal initial stump dressing using cotton wool, Netelast, and no tape. A poor crepe can occlude venous drainage and produce stump oedema.
Most lower limb amputations are now done because of vascular insufficiency. In 1987, 4985 new lower limb amputees were referred to the disablement service centres (previously known as artificial limb and appliance centres) in England. Two thirds of these amputations were done for peripheral vascular disease and more than a fifth for the complications of diabetes mellitus. About $80 \%$ of the patients were over 60 years old at the time of amputation. The problems to be faced after amputation, therefore, are not only those of achieving independent mobility, but also of managing illnesses associated with advancing years and returning to the community.

There are three important aims:

- To produce a soundly healed stump and a patient who can walk. The level of amputation is therefore crucial: above the knee amputations heal readily but many patients who have them never master walking on a prosthesis. The converse is true of below knee amputations

- Early rehabilitation with a prosthesis

- Cost effectiveness.

These may be achieved with a hospital based rehabilitation team that liaises closely with the disablement service centre, the general practitioner, and the community services.

\section{Before the stump has healed}

Early referral of a patient with critical ischaemia of the lower limb to a specialist vascular surgeon is essential so that either salvage of the limb by reconstructive surgery or interventional radiology may be attempted, and (if these are not possible) sufficient time will be available to prepare the patient for amputation.

Compression bandaging should not be used on the stump during the early postoperative period while it is healing. The patient should be mobilised by using the pneumatic post amputation mobility aid (PPAM aid) under the supervision of an experienced physiotherapist. Initially, he or she should use parallel bars for support, and then graduate to elbow crutches. The patient will then be referred to the local disablement service centre to be assessed and measured for a prosthesis, unless it is thought that walking is unlikely, in which case a wheelchair will be provided.

\section{Mobilisation}

Those who can walk are taught how to cope. The Department of Health has produced a booklet called Your next step forward. It is an introduction to the disablement service centre, and gives information about the services available and how to care for the stump and the artificial limb. Good communication between the hospital, the disablement service centre, and the general practitioner is essential.

When the stump is soundly healed the patient will receive his or her first artificial limb-usually a definitive prosthesis with a temporary socket. Occasionally a temporary or pylon limb may be used-for example, for patients who are frail and require the lightest possible prosthesis, or for those in whom there is doubt about whether they will use a prosthesis. During this rehabilitation phase patients are shown how to care for the 
Once the suture line has healed a pneumatic postamputation mobility aid is excellent for early walking.

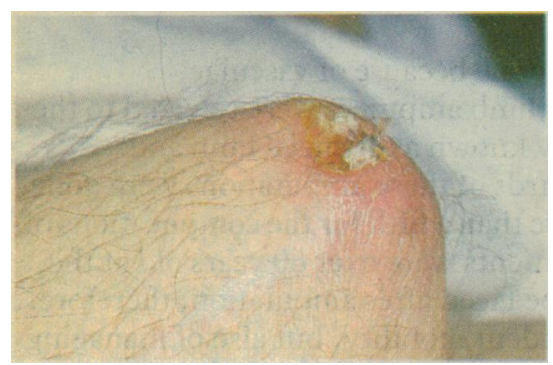

Poor above knee stump with retraction of muscle and erosion of bone through the skin.

\section{The amputated limb}

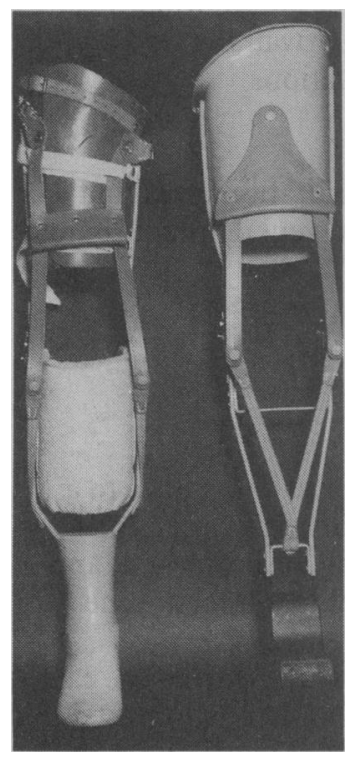

A below knee (left) and above knee (right) rocker pylon that can be used while the final prosthesis is being built.

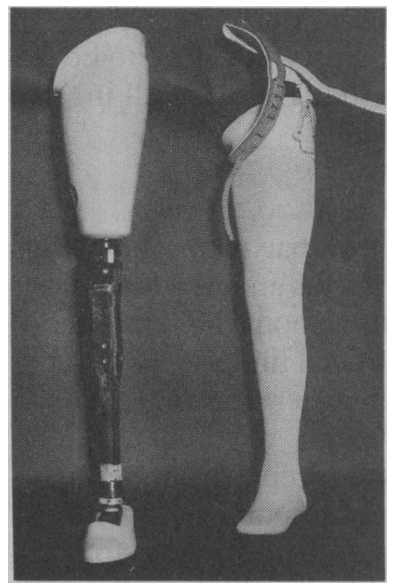

An Endolite above knee prosthesis with suction socket at the fitting stage and with the external cover and rigid pelvic strap. stump, put on the artificial limb, and dress. The length of time they spend standing is increased, and they learn to walk - at first on the flat and later on sloping and uneven surfaces - and to pick up objects from the floor and to climb stairs. They are taught how to pick themselves up after falling over. Close supervision is therefore essential. The occupational therapist and social worker will by this time have visited their homes and assessed what structural changes will be required or if reaccommodation is necessary.

Progress is discussed at regular meetings of the rehabilitation team.

Before discharge from hospital not only must the patients be independently mobile, but also the place to which they are going (their own home, or the home of family or friends) must be prepared. The community servicesprovided by the district nurse, the home help, and "meals on wheels"should be arranged before discharge, when close liaison with the general practitioner is vital so that continuity of care and support are maintained. The general practitioners should be in a position to smooth over any of the initial fears that the families may have about accepting amputees back into the home.

\section{After discharge from hospital}

Amputation causes a considerable change in body symmetry, which will affect both the posture and the body image. Most patients seem to adjust well to life with an artificial limb, though there are three topics on which medical advice and help may be sought: the amputated limb, general health, and social activities.

\section{The stump}

The stump is a vulnerable area that requires great care and attention.

General care-An amputee will have been taught how to care for the stump (including simple hygienic measures) in the hospital and at the disablement service centre. The stump usually takes a few months to mature, so that in the early period after discharge from hospital it should be examined regularly for oedema and infection. Swelling should be controlled by stump compression socks. Bandages should be applied to the stump only by those skilled in the technique. Infection should be treated with rest and antibiotics. Stump socks are provided, and the choice of material is decided by the fit of the limb to avoid chaffing and not by the patient's preference. Problems with the weight bearing areas of the stump and the interface of the prosthesis will arise as the stump shrinks and the socket becomes loose. An amputee should be advised to wear more than one stump sock, and an appointment should be made at the disablement service centre for adjustment of the socket.

Stump pain-Neuromas may cause pains varying from dull aches to electric shocks. Transcutaneous nerve stimulation may relieve the symptoms, but excision may be required. Pain may also be caused by osteomyelitis, the growth of a bony spur, or ischaemia. Radiographs will confirm the diagnosis of the first two, but if ischaemia is suspected an urgent appointment with the surgeon is indicated. Pain from the lumbar spine may result from tilting of the pelvis because the artificial limb is too short or because the gait is unsatisfactory. In such cases the amputee should be referred to the disablement service centre for treatment.

\section{Phantom limb pain}

Most patients experience phantom pains, which can be depressing. Reassurance, carbamazepine, transcutaneous nerve stimulation, tapping the stump, and acupuncture have all been tried with varying degrees of success, but the symptoms may become blunted with time. Carbamazepine is usually the first line of treatment and is often effective.

\section{Discomfort from the prosthesis}

Discomfort from the buckles and straps of the prosthesis, and generalised aches and pains associated with the use of the prosthesis are common. An above the knee amputee may be given a prosthesis that has a waistband and shoulder strap, which may cause pain from chaffing and pressure. One simple cure is to pad out the straps. Alternatively, the doctor at the disablement service centre may prescribe a suction socket, thereby 


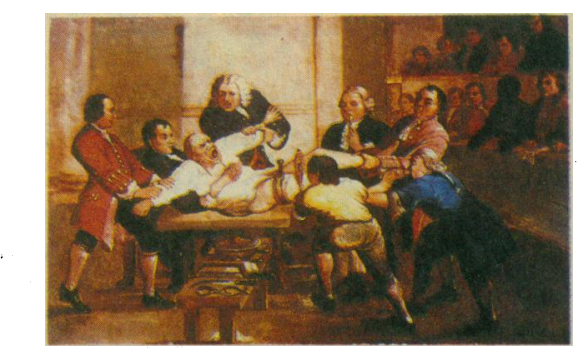

"The horror of surgery must be minimised by careful counselling." An amputation performed without anaesthesia at St Thomas's Hospital in the nineteenth century.

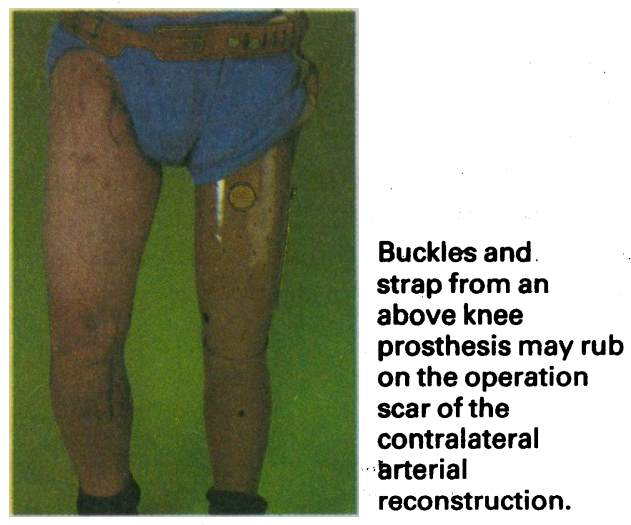

\section{Social activities}

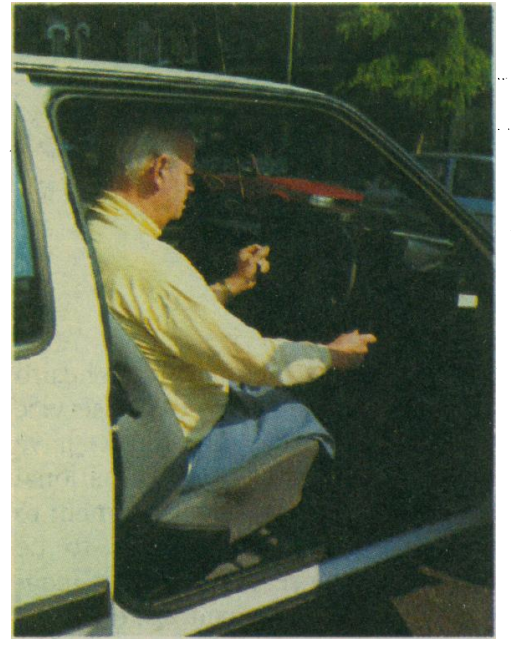

Bilateral above knee amputation does not preclude driving and independence.

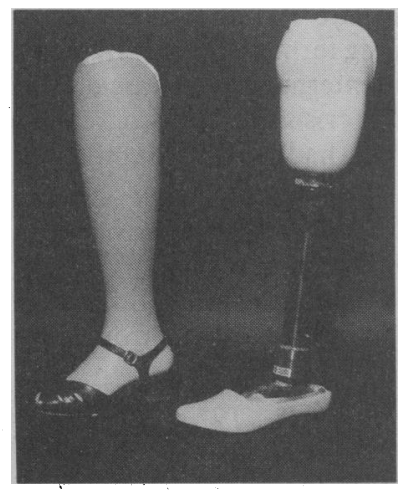

An Endolite supracondyle patella bearing prosthesis in the fitting stage (right) and with external cover (left). removing the necessity for straps. As most of the patients are elderly, however, their stumps and general health usually preclude the use of suction sockets. Where possible analgesics (which may cause constipation) should be avoided. Instead regular physiotherapy or osteopathy should be prescribed.

\section{Psychological effects}

Loss of a limb has been described in the same emotional terms as a bereavement; these feelings are intensified by phantom limb sensations, and are more pronounced in elderly people. If the explanation of why the limb was amputated has not been accepted by the patient he or she may blame the medical staff for the disability. This is not always a hostile reaction and may be expressed as a demand for extra attention. Psychiatric help may occasionally be needed.

\section{Care of the artificial limb}

This is entirely within the province of the disablement service centres, which offer a service throughout the United Kingdom even when the amputee is away from home. If damage or breakdown occurs the patient may telephone the local disablement service centre to arrange an appointment.

\section{General health of the amputee}

Although the long term prognosis of patients who have had amputations for peripheral vascular disease is poor, it is essential to maintain their dignity and health. Problems of aging (such as prostatic hypertrophy causing nocturia) need prompt treatment. Attention must be paid to controlling conditions that may have a deleterious effect on the stump, the remaining limbs, and mobility - for example, diabetes mellitus, cardiorespiratory disease, and arthritis. Clearly it is imperative that every effort is made to prevent irreversible ischaemia in the remaining leg. If a new condition is diagnosed the disablement service centre should be informed.

\section{Work}

Amputees who wish to return to work should be encouraged to do so. If they are unable to take up their original employment the disablement resettlement officer should be contacted.

\section{Driving}

Amputees must inform the Driver and Vehicle Licensing Centre (DVLC) and their insurance company about their disability, but the amputation will not debar them from driving as the car can be modified. The licensing centre stipulates that automatic transmission is preferable. For a bilateral amputee hand controls are required. The amputee should be advised to contact the British School of Motoring or a local agency recommended by the disablement service centre for a trial assessment and should join the Disabled Drivers' Association, members of which will give practical advice.

\section{Public transport}

Getting on or off buses and escalators should initially be supervised by an occupational therapist. Many elderly amputees never manage without help.

\section{Shoes}

The soles and heels of any new shoes require close scrutiny. When the heel is flat on the ground the sole must be as well otherwise the gait may be altered. The disablement service centre can adjust the height of a prosthesis if the patient wishes to use heels of different heights, and there is an adjustable heel device that can be fitted to many limbs so that the patients may adjust the height of the heel themselves.

\section{Leisure activities}

After spending some time in hospital most patients gain weight. Amputees should therefore be encouraged to take up some form of physical exercise.

\section{Holidays}

Amputees should be advised to take crutches when they travel away from home in case of mechanical failure of the limb. If spending their holidays within the United Kingdom they should obtain the telephone number of the nearest disablement service centre, which will always carry out emergency repairs; if going abroad they should make inquiries about reciprocal repair arrangements. 
The artificial limb and appliance centres were criticised in a report published in 1987 to the Department of Health by a committee chaired by Lord McColl. The committee suggested that the service could be improved by raising the technical standards of amputation; by setting up specialist multidisciplinary hospital teams that would work in close cooperation with the medical staff at the centres and take an active part in rehabilitation; by giving more responsibility to prosthetists and encouraging them to be responsible for continuing care; and, finally, by restructuring of management. Some changes have already been implemented-such as the integration of the service into health regions with integrated budgets. Prosthetists may now tender for provision and service contracts, thus allowing them to become independent of the major suppliers of artificial limbs.

The demands of amputees on the medical and associated services will vary according to their independence. Some will need little medical help; a new elderly amputee, however, will require extensive support and supervision if he or she is to be discharged from an acute hospital and return to the community. After discharge from hospital the general practitioner will not only look after the immediate physical requirements of the amputee but also coordinate the community support, the hospital services, and the services of the disablement service centre.

Only I Known That a Year Ago..... published by RADAR. This booklet reviews what is available to disabled people, their families, and friends and includes the addresses of many useful organisations and associations

We thank Mr Kiril Gray and Mr Ray Holland (BLESMA) for their help, and Dr Linda Marks of the DSA limb fitting centre, Stanmore, for her criticisms and comments. We are grateful to the Royal College of Surgeons of England for permission to reproduce the picture of the amputation in the eighteenth century, and we acknowledge with thanks the assistance of the audiovisual department, St Mary's Hospital, London, in the preparation of the illustrations.

Mr S J D Chadwick, FRCs, is consultant surgeon, Northwick Park Hospital, Harrow, and Mr John H N Wolfe, FRCs, is consultant vascular surgeon, St Mary's Hospital, London.

The ABC of Vascular Diseases has been edited by Mr John $\mathrm{H}$ N Wolfe.

This is the second in a series of articles responding to the questions raised by the BMA's document, "Leading for Health." The document looks well beyond the coming British election and raises questions about health and health care that will be on the agenda of many countries into the next century.
Lancaster Health

Authority, Lancaster Moor Hospital, Lancaster

LA1 3JR

Professor Alwyn Smith, FFPHF, chairman

\title{
Leading for Health
}

\section{Setting a strategy for health}

\author{
Alwyn Smith
}

The international debate on the need for national health strategies and what they should contain has been proceeding for several decades without significantly attracting the involvement of the medical profession in the United Kingdom. Indeed, the worldwide crisis of confidence in traditional attitudes to the form and content of professional health practice has often seemed irrelevant to British doctors because of our complacent satisfaction with the NHS and our consequent belief that we have little need to be concerned. This attitude has changed with the government's health reforms, and in Leading for Health ${ }^{1}$ the BMA has set an agenda for discussions on health and policy setting for the 1990s.

\section{The health crisis}

When the NHS was introduced parliamentary and public debate identified a set of objectives more far reaching than the simple one of providing medical care free at the time of use. These were to promote the nation's health; to ensure the equitable distribution of health care; to render the health service accountable to the nation; and to invest the activities and development of the NHS with a sense of purpose. It is disappointing after more than 40 years to examine its record of achievement against these objectives. The nation's health has progressed less well than that of many other countries; there remain serious inequalities in health and access to health care; accountability of the service to the electorate has almost disappeared; and we have only just begun to develop a strategy for the national health. However, the conversion of the government to the notion of a national health strategy is to be welcomed enormously, and the responses of the main opposition parties and of the BMA encourage the hope that the long overdue debate on the politics of health is about to begin. The pursuit of the national health is as important a matter for political debate as the national economy or the defence of the realm.

It is not only in the United Kingdom that there is a preoccupation with health strategies; indeed, we have come to the subject quite late and after many other countries have been pursuing formally stated strategies for a decade or more. The international public debate arose from a mid-century crisis in the progress of public health services that had several identifiable origins:

- Reduced mortality and a reduced birth rate have led to populations with a larger proportion of elderly people, which has in turn halted the crude decline in mortality and increased the prevalence of chronic and often intractable disease

- Demand for health care has grown and most countries have found it politically difficult to match this growth with a commensurate increase in supply 